Case Report

\title{
Spontaneous Knee Ankylosis through Heterotopic Ossification after Total Knee Arthroplasty
}

\author{
Samuel Boulezaz, ${ }^{1}$ Emmanuel Gibon, ${ }^{1}$ Philippe Loriaut, ${ }^{2}$ Laurent Casabianca, ${ }^{2}$ \\ Romain Rousseau, ${ }^{2}$ Benjamin Dallaudiere, ${ }^{3,4}$ and Hugues Pascal-Moussellard ${ }^{2}$ \\ ${ }^{1}$ Department of Reconstructive and Orthopaedic Surgery, Clinical Orthopaedic Research Centre, Université Paris Descartes, \\ Hôpital Cochin, 27 rue du Faubourg Saint Jacques, 75014 Paris, France \\ ${ }^{2}$ Department of Orthopaedic and Sport Surgery, Pitié Salpêtrière Hospital, Pierre and Marie Curie University, \\ 47 boulevard de l'Hôpital, 75013 Paris, France \\ ${ }^{3}$ Centre d'Imagerie Ostéo-Articulaire, Clinique du Sport de Bordeaux-Mérignac 2, rue Négrevergne, \\ 33700 Mérignac, France \\ ${ }^{4}$ Département d’Imagerie Musculo-Squelettique, Centre Hospitalier Universitaire Pellegrin, Place Amélie Léon Rabat, \\ 33000 Bordeaux, France
}

Correspondence should be addressed to Samuel Boulezaz; samuel.boulezaz@gmail.com

Received 5 November 2015; Revised 3 February 2016; Accepted 14 February 2016

Academic Editor: Charbel D. Moussallem

Copyright (C) 2016 Samuel Boulezaz et al. This is an open access article distributed under the Creative Commons Attribution License, which permits unrestricted use, distribution, and reproduction in any medium, provided the original work is properly cited.

\begin{abstract}
This paper reports on a case of total ankylosis of the knee after a cruciate-sacrificing cemented total knee arthroplasty (TKA). An 82year-old female patient previously underwent primary TKA for osteoarthritis twenty years ago in our institution. She had recovered uneventfully and returned to her regular activities. There was no history of postsurgical trauma; however, she progressively lost knee range of motion. Radiographs revealed severe bridging heterotopic ossification.
\end{abstract}

\section{Introduction}

Heterotopic ossification ( $\mathrm{HO}$ ) is the abnormal formation of mature lamellar bone in soft tissues and is a common finding in total hip arthroplasty; however, it is an unusual phenomenon after total knee arthroplasty (TKA). An incidence of $1 \%$ to $42 \%$ has been reported in the literature [1-8].

HO after TKA rarely results in complications. Most patients are asymptomatic, but some have reported pain or limited range of motion. HO leading to a bony ankylosis after TKA is very uncommon. Very few cases of total bony ankylosis after TKA have been reported, most of which had a history of previous sepsis [1, 4, 9-12].

The purpose of this case report is to describe the clinical and radiological features of spontaneous knee ankylosis by HO following primary TKA for osteoarthritis.

\section{Case Report}

In November 1994, a 62-year-old woman underwent a cemented left TKA in our institution using a G2S (FII, SaintEtienne, France) posterior stabilized total knee prosthesis for primary osteoarthritis. Her only reported medical comorbidities at this time included hypertension (treated) and an increased body mass index (BMI) of $29.3(75 \mathrm{~kg}, 160 \mathrm{~cm})$.

Preoperatively, the patient was very disabled by her knee pain. Her walking tolerance was less than a hundred meters. She had been using analgesia and had received several knee infiltrations that were of limited efficacy prior to surgical management. The reported range of motion of the affected knee was 90/10/0 preoperatively. Her TKA was performed without a tourniquet and through a medial parapatellar approach. 


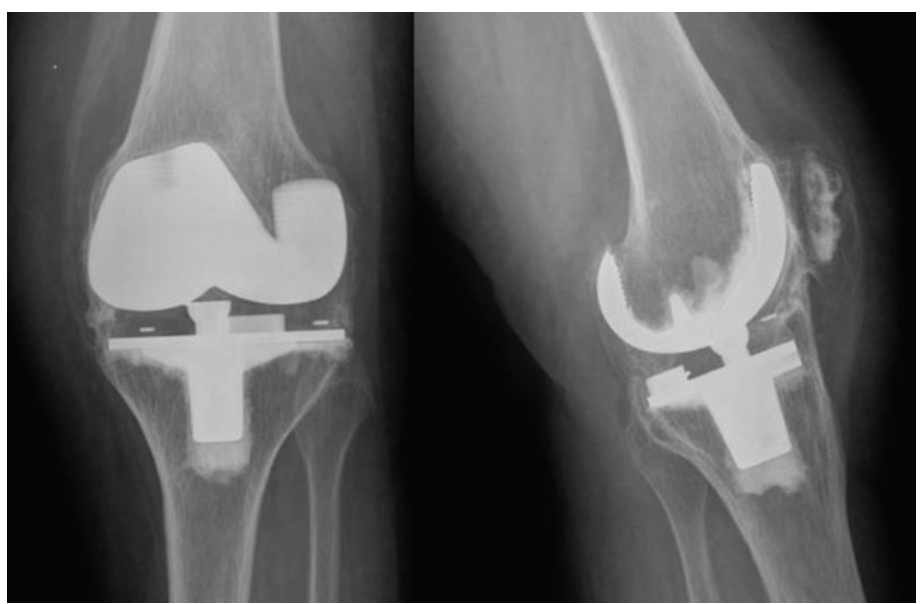

FIGURE 1: AP and lateral X-rays showing massive heterotopic ossification around the implants.

Her infrapatellar fat pad was partially resected. As there was no major frontal deviation there was no need for ligament release. All the implants (tibial, femoral, and patellar) were cemented. No complications were reported during surgery.

Postoperatively, there was no malalignment or malposition on the X-rays, and she recovered uneventfully. Reported range of motion to her knee was 100/10/0 at 6-week followup. No further clinical data was available for this patient as she returned to her country of origin.

She returned to the clinic 20 years later, at 82 years of age. Clinical examination revealed a knee locked in full extension and quadriceps atrophy. The patient had no significant trauma history and reported no knee pain. Global function and autonomy were preserved. X-ray radiographs displayed massive circumferential and complete extra-articular ossifications around her prosthesis. The ossifications were located in both the femorotibial joint space and the femoropatellar joint. There was neither intra-articular calcification nor joint effusion (Figure 1).

A CT scan confirmed the above observations and showed complete circumferential ankylosis with an absence of osteolysis around the TKA with a posterior joint fusion (Figures 2 and 3). Cancellous bone weft was with aspect of demineralized age related (82 years old) with no malignant or Paget disease signs. A complete quadriceps amyotrophy was observed (Figure 4). There was neither fibrocartilage calcification nor hyaline cartilage lesions suggesting chondrocalcinosis or gout. No abarticular deformation or sign of renal osteodystrophy was observed.

In the 20 years where there was no documented orthopedic follow-up, the patient did not recall any trauma, infiltration, or infection in her knee. The scar remained clean.

Final radiological diagnosis was spontaneous total knee ankylosis with HO. Indeed, this complete knee ossification could be compared to dystrophic ossification leading to ankylosis after total hip arthroplasty.

Investigations focused on etiological research. Blood tests and blood cultures showed no evidence of infection or an inflammatory syndrome. Nevertheless, the hypothesis of a chronic infection was explored.

A CT-guided articular puncture was consequently performed and cultures were negative. Further surgery was offered to the patient, but she declined.

\section{Discussion}

This case report provides insight on a very rare situation of total knee ankylosis after TKA showing that HO may severely alter knee range of motion.

$\mathrm{HO}$ can be detected on a bone scan as early as three weeks postoperatively, with increased uptake in the soft tissues. Plain radiographs are negative for four to six weeks. However, the increased bone turnover that occurs in $\mathrm{HO}$ can be detected as early as one week after surgery, with excessive increase in the specific osteoclastic and osteoblastic markers (CTX-1 and P1NP) detected in venous blood. Extensive bone formation may occur within three months, but full maturation of bone takes up to one year $[3,13]$.

Risk factors of $\mathrm{HO}$ around the knee have been widely studied. PCL reconstruction in multiligament knee injuries [14], long-term sedation [15], dislocation, high Injury Severity Score, and closed head injury [16] have been found to be risk factors in native knee. Osteoarthritis, wound healing problems, hypertrophic arthritis, notching [17], elevated BMI, male gender [18], preoperative grade of osteophyte formation [19], limited postoperative knee flexion, excessive periosteal trauma, postoperative forced manipulation [20], postoperative effusion [21], and increased lumbar bone mineral density [5] have been found to be risk factors in total knee arthroplasty. The patient in this case presented with only limited postoperative knee flexion and an elevated BMI in her risk factor profile.

Several treatment options for symptomatic $\mathrm{HO}$ have been discussed in the literature. Physical therapy is the main recommended treatment [22]. Some authors have proposed surgical excision of large symptomatic $\mathrm{HO}$ while others 


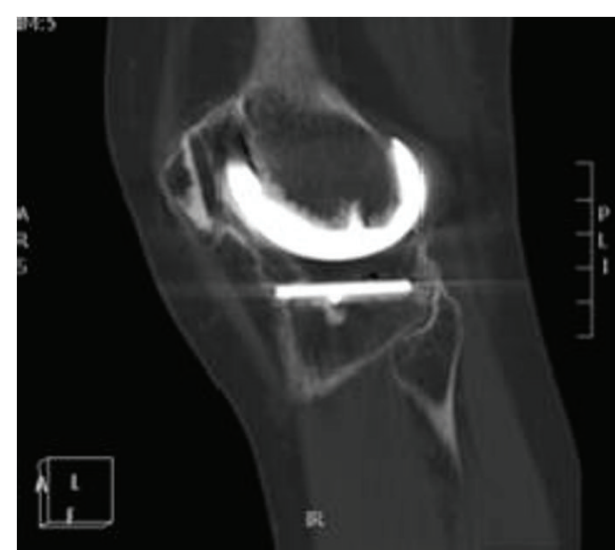

(a)

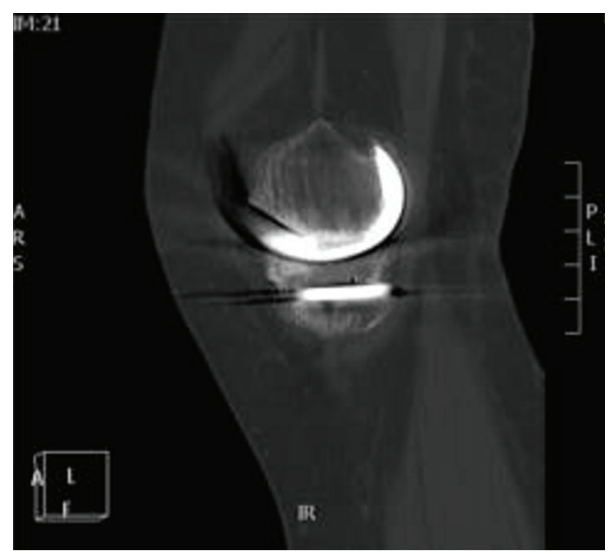

(c)

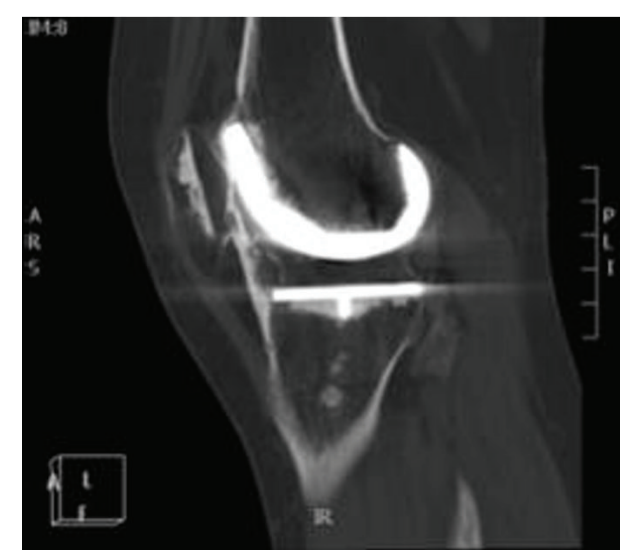

(b)

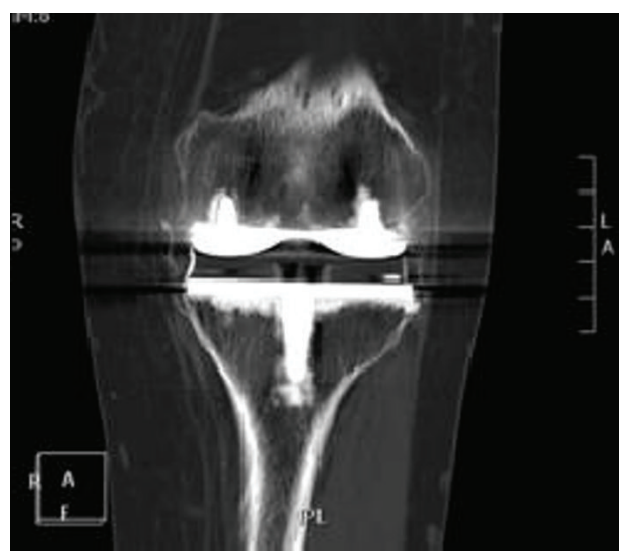

(d)

FIgURE 2: CT scan views showing a circumferential complete ankylosis of the knee.

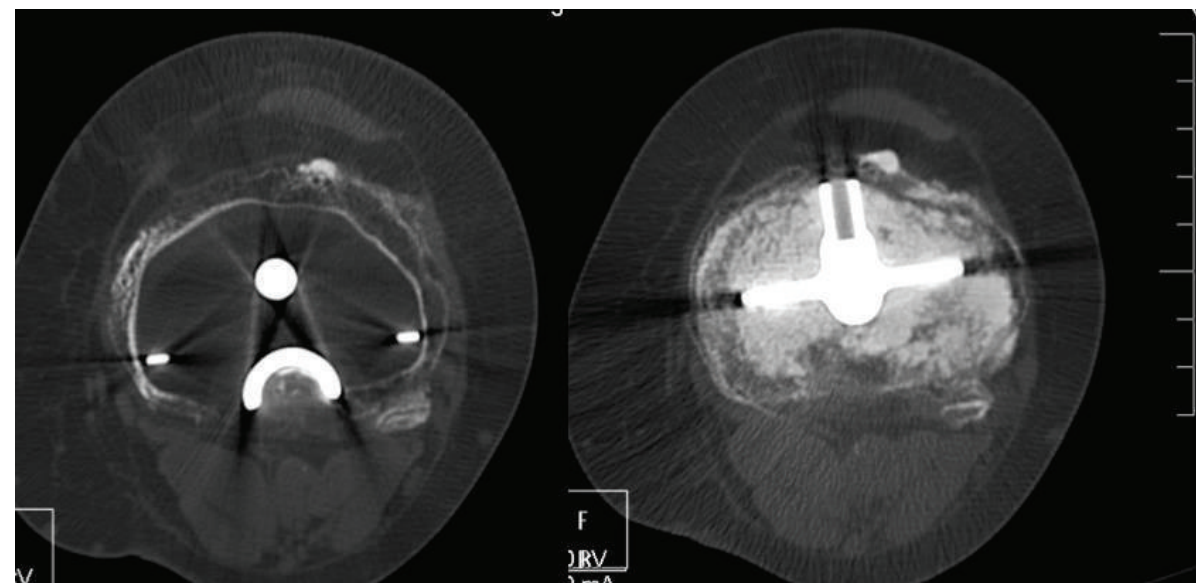

FIGURE 3: No lysis between cement and cancellous bone.

suggested resection with concurrent manipulation, followed by postoperative indomethacin for 2 months and physical therapy [21-23].

Several preventive measures have been discussed in the literature. Irradiation in the prevention of $\mathrm{HO}$ has been discussed but is controversial as it carries significant side effects. Nonsteroidal anti-inflammatories (NSAIDs) such as indomethacin have inconclusive results and increase the risk of major bleeding $[3,24,25]$.

A simple but effective measure is to wash the joint with serum during the surgical procedure. This should reduce the presence of bone particles in periarticular soft tissues. 


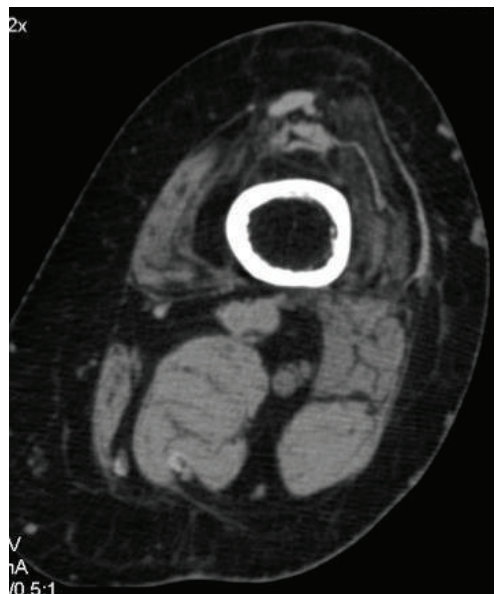

FIGURE 4: Complete quadriceps amyotrophy.

\section{Conclusion}

Complete ankylosis after total knee arthroplasty is a rare but significant complication; physicians who care for arthroplasty patients should be made aware of.

\section{Competing Interests}

The authors declare that they have no competing interests regarding the publication of this paper.

\section{Acknowledgments}

The authors thank Jessica Goldman and Dina Popovic who helped in editing this paper.

\section{References}

[1] A. L. Dodds and G. C. Keene, "Severe heterotopic ossification following total knee replacement," Case Reports in Orthopedics, vol. 2014, Article ID 265489, 3 pages, 2014.

[2] F. Atamaz, S. Aydogdu, S. Hepgüler, and H. Sur, "Heterotopic ossification after total knee arthroplasty," Acta Orthopaedica et Traumatologica Turcica, vol. 40, no. 3, pp. 202-206, 2006.

[3] T. N. Board, A. Karva, R. E. Board, A. K. Gambhir, and M. L. Porter, "The prophylaxis and treatment of heterotopic ossification following lower limb arthroplasty," The Journal of Bone \& Joint Surgery - British Volume, vol. 89, no. 4, pp. 434-440, 2007.

[4] J. Cook and R. D. Scott, "Bony ankylosis following total knee arthroplasty: a case report," Journal of Arthroplasty, vol. 20, no. 1, pp. 122-124, 2005.

[5] J. P. Furia and V. D. Pellegrini Jr., "Heterotopic ossification following primary total knee arthroplasty," The Journal of Arthroplasty, vol. 10, no. 4, pp. 413-419, 1995.

[6] J. E. Lovelock, H. J. Griffiths, A. M. Silverstein, and P. S. Anson, "Complications of total knee replacement," American Journal of Roentgenology, vol. 142, no. 5, pp. 985-992, 1984.

[7] T. Sterner, G. Saxler, and B. Barden, "Limited range of motion caused by heterotopic ossifications in primary total knee arthroplasty: a retrospective study of 27/191 cases," Archives of
Orthopaedic and Trauma Surgery, vol. 125, no. 3, pp. 188-192, 2005.

[8] D. Tigani, A. Marinelli, G. Bianchi, P. Trentani, F. Trentani, and D. Antonioli, "Heterotopic ossifications subsequent to knee arthroplasty," La Chirurgia Degli Organi di Movimento, vol. 89, no. 2, pp. 135-141, 2004.

[9] Y.-H. Kim, S.-H. Cho, and J.-S. Kim, "Total knee arthroplasty in bony ankylosis in gross flexion," The Journal of Bone \& Joint Surgery-British Volume, vol. 81, no. 2, pp. 296-300, 1999.

[10] T. Ojima, A. Yokogawa, K. Yamakado et al., "Bilateral total knee arthroplasty after spontaneous osseous ankylosis in rheumatoid arthritis," Modern Rheumatology, vol. 15, no. 2, pp. 139-143, 2005.

[11] E. Thienpont, T. Schmalzried, and J. Bellemans, "Ankylosis due to heterotopic ossification following primary total knee arthroplasty," Acta Orthopaedica Belgica, vol. 72, no. 4, pp. 502 506, 2006.

[12] P. K. Chan, K. Y. Chiu, F. Y. Ng, and C. H. Yan, "Bony ankylosis of the knee secondary to heterotopic ossification after total knee arthroplasty: a case report," Journal of Orthopaedic Surgery (Hong Kong), vol. 22, no. 3, pp. 434-436, 2014.

[13] J. M. Wilkinson, I. Stockley, A. J. Hamer, N. A. Barrington, and R. Eastell, "Biochemical markers of bone turnover and development of heterotopic ossification after total hip arthroplasty," Journal of Orthopaedic Research, vol. 21, no. 3, pp. 529-534, 2003.

[14] D. B. Whelan, A. P. Dold, T. Trajkovski, and J. Chahal, "Risk factors for the development of heterotopic ossification after knee dislocation," Clinical Orthopaedics and Related Research, vol. 472, no. 9, pp. 2698-2704, 2014.

[15] A. Sugita, J. Hashimoto, A. Maeda et al., "Heterotopic ossification in bilateral knee and hip joints after long-term sedation," Journal of Bone and Mineral Metabolism, vol. 23, no. 4, pp. 329332, 2005.

[16] W. J. Mills and N. Tejwani, "Heterotopic ossification after knee dislocation: the predictive value of the Injury Severity Score," Journal of Orthopaedic Trauma, vol. 17, no. 5, pp. 338-345, 2003.

[17] K. E. Roth, G. Salzmann, G. S. Maier, I. Schmidtmann, J. D. Rompe, and K. Babin, "Risk factors for heterotopic ossification and spur formation after total knee arthroplasty," Archives of Orthopaedic and Trauma Surgery, vol. 134, no. 7, pp. 991-996, 2014.

[18] D. F. Dalury and W. A. Jiranek, "The incidence of heterotopic ossification after total knee arthroplasty," The Journal of Arthroplasty, vol. 19, no. 4, pp. 447-452, 2004.

[19] T. Toyoda, H. Matsumoto, T. Tsuji, J. Kinouchi, and K. Fujikawa, "Heterotopic ossification after total knee arthroplasty," The Journal of Arthroplasty, vol. 18, no. 6, pp. 760-764, 2003.

[20] R. Iorio and W. L. Healy, "Heterotopic ossification after hip and knee arthroplasty: risk factors, prevention, and treatment," The Journal of the American Academy of Orthopaedic Surgeons, vol. 10, no. 6, pp. 409-416, 2002.

[21] M. Hasegawa, T. Ohashi, and A. Uchida, "Heterotopic ossification around distal femur after total knee arthroplasty," Archives of Orthopaedic and Trauma Surgery, vol. 122, no. 5, pp. 274-278, 2002.

[22] J. Pham and R. Kumar, "Heterotopic ossification after total knee arthroplasty," The American Journal of Orthopedics, vol. 26, no. 2, pp. 141-143, 1997.

[23] E. L. Freedman and D. M. Freedman, "Heterotopic ossification following total knee arthroplasty requiring surgical excision," American Journal of Orthopedics, vol. 25, no. 8, pp. 559-561, 1996. 
[24] J. Bellemans, P. Claerhout, T. Eid, and G. Fabry, "Severe heterotopic ossifications after total knee arthroplasty," Acta Orthopaedica Belgica, vol. 65, no. 1, pp. 98-101, 1999.

[25] W. Braun, "Irradiation for the prevention of heterotopic ossification following surgery of the hip and knee joint. Report of initial experiences," Der Chirurg, vol. 60, no. 11, pp. 795-800, 1989. 


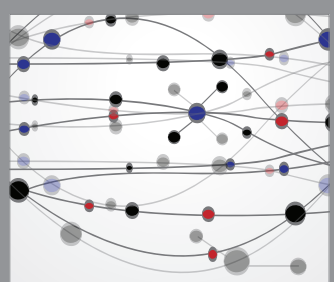

The Scientific World Journal
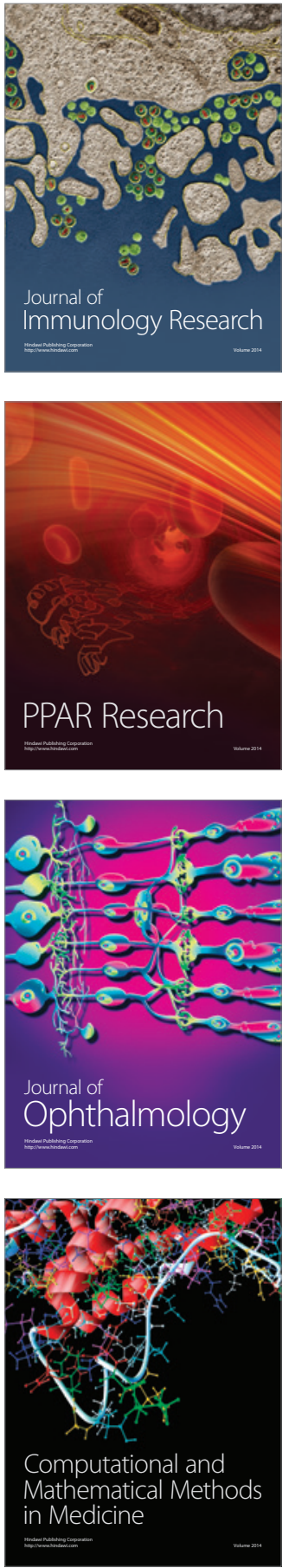

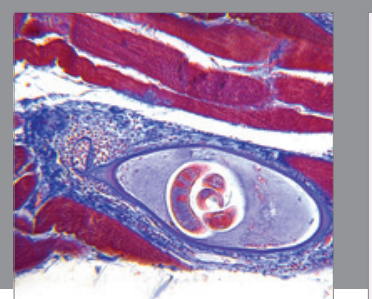

Gastroenterology Research and Practice

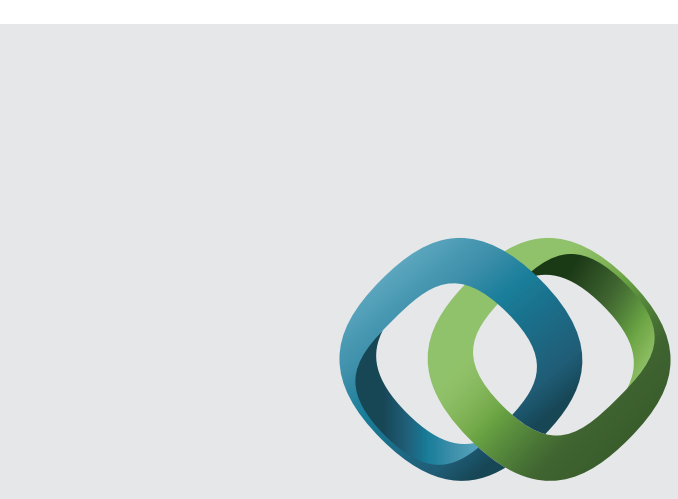

\section{Hindawi}

Submit your manuscripts at

http://www.hindawi.com
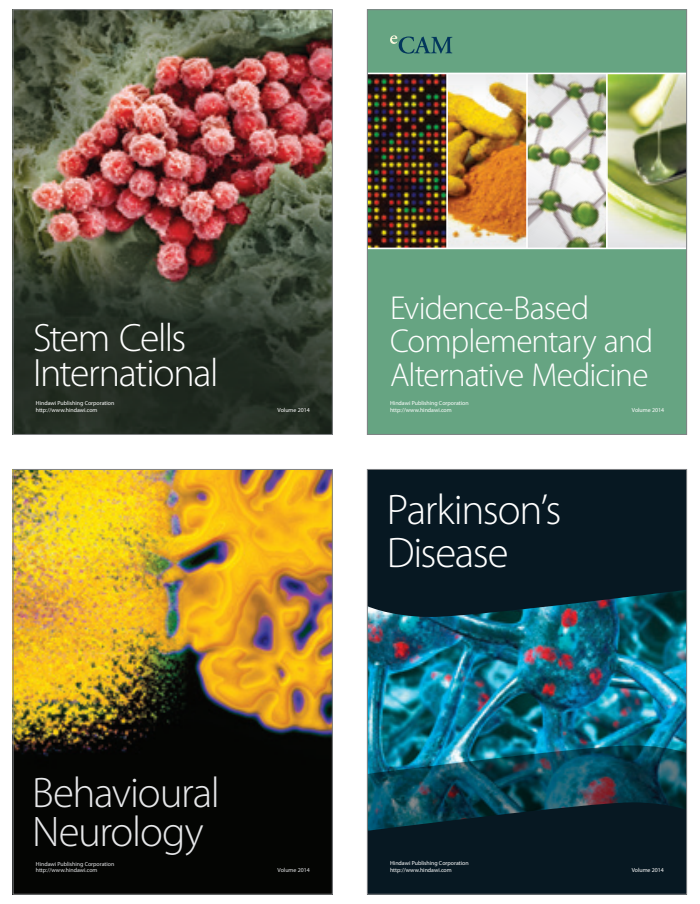
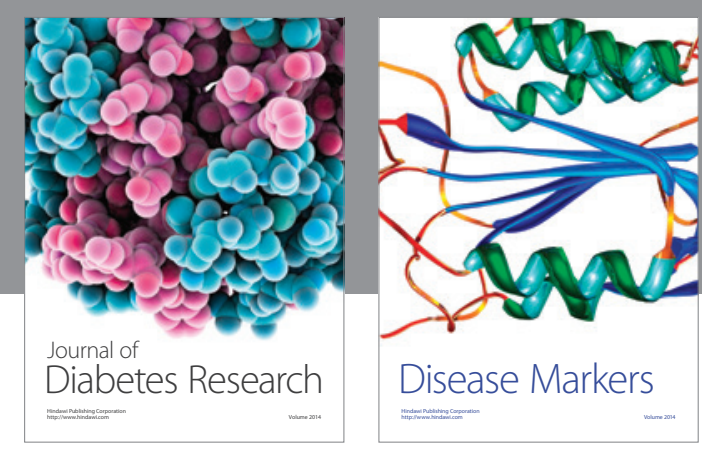

Disease Markers
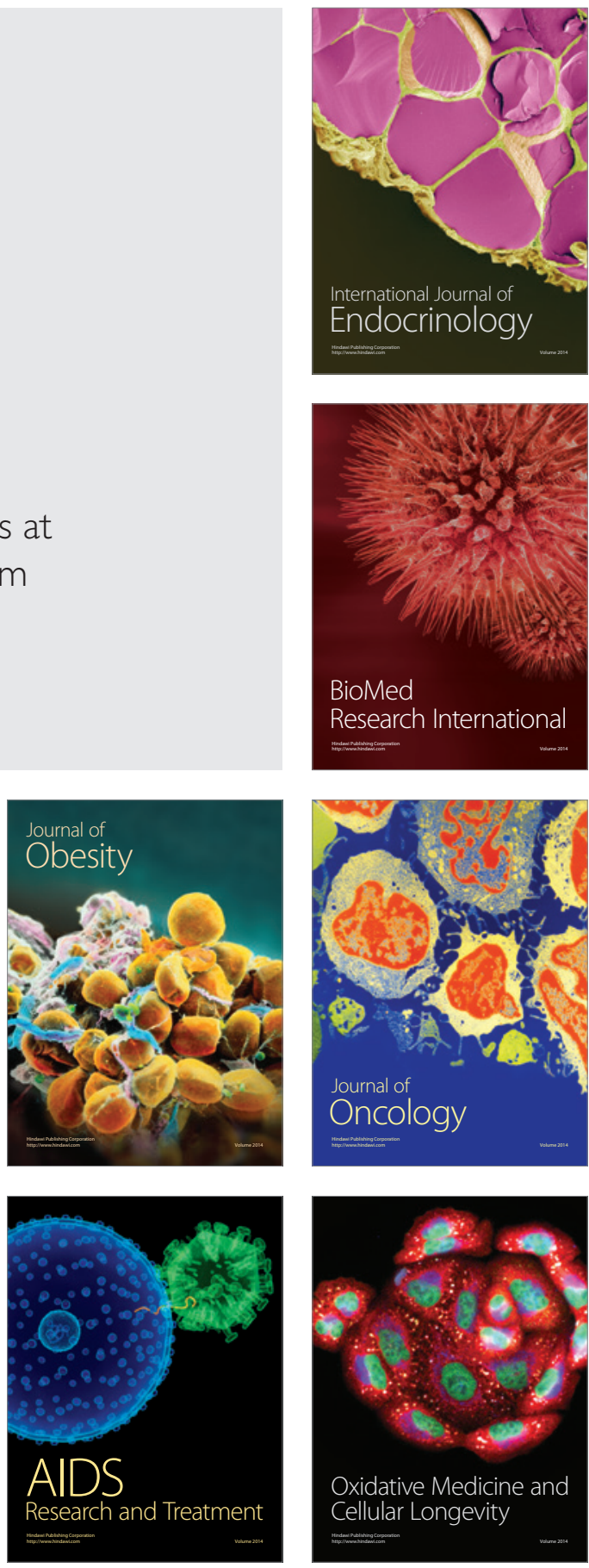